\title{
STATUS OF THE HADRONTHERAPY PROJECTS IN EUROPE
}

\author{
Jean-Michel Lagniel, GANIL, Caen, France
}

\section{Abstract}

Robert R. Wilson, an accelerator pioneer working with E.O. Lawrence at Berkeley, made the first proposal to treat cancer using proton beams more than 60 year ago (1946). In a first period, many accelerators built for nuclear and particle physics research have been progressively used to treat patients all over the word. It was the time of the hadrontherapy "first generation". As it has been the case for the evolution of the synchrotron radiation sources, starting with Loma Linda in 1990, a "second generation" of dedicated facilities has then been built to treat cancer without the constraints imposed to "parasitic users". The total number of patients treated with protons was about 10.000 in 1993 and 50.000 in 2006, the progression looks to be exponential. Hadrontherapy is today part of the medical business landscape, several companies sell turnkey protontherapy centres and the fight is started for carbon centres. The status of some hadrontherapy projects in Europe (existing facilities and future projects) as well as the industrial aspects will be presented in this framework.

\section{INTRODUCTION}

The particle therapy facilities in operation are listed below (from PTCoG [1]). In this table the fist column is the first year of operation, the following are the type of beam $(p=$ protons, $i=$ ions $)$, the location and the number of treated patients (see [1] for detailed information).

\begin{tabular}{lll}
1961 & $\mathrm{p}$ & Harvard, Boston, USA \\
1969 & $\mathrm{p}$ & ITEP, Moscow, Russia \\
1975 & $\mathrm{p}$ & St.Petersburg, Russia \\
1979 & $\mathrm{p}$ & Chiba, Japan \\
1983 & $\mathrm{p}$ & PMRC1, Tsukuba, Japan \\
1984 & $\mathrm{p}$ & PSI-1, Villigen, Switzeland \\
1989 & $\mathrm{p}$ & Dubna, Russia \\
1989 & $\mathrm{p}$ & Uppsala, Sweden \\
1989 & $\mathrm{p}$ & Clatterbridge, England \\
1990 & $\mathrm{p}$ & Loma Linda, CA, USA \\
1991 & $\mathrm{p}$ & Nice, France \\
1991 & $\mathrm{p}$ & CPO, Orsay, France \\
1993 & $\mathrm{p}$ & iThemba, South Africa \\
1993 & $\mathrm{p}$ & MPRI, IN, USA \\
1994 & $\mathrm{p}$ & UCSF, CA, USA \\
1994 & $\mathrm{i}$ & HIMAC, Chiba, Japan \\
1995 & $\mathrm{P}$ & TRIUMF, Canada \\
1996 & $\mathrm{p}$ & PSI-2, Villigen, Switzeland \\
1997 & $\mathrm{i}$ & GSI, Germany \\
1998 & $\mathrm{p}$ & HMI, Berlin, Germany \\
1998 & $\mathrm{p}$ & NCC, Kashiwa, Japan \\
2001 & $\mathrm{p}+\mathrm{i}$ & HIBMC,Hyogo, Japan \\
2001 & $\mathrm{p}$ & PMRC2, Tsukuba, Japan \\
2001 & $\mathrm{p}$ & NPTC, Boston, USA \\
2002 & $\mathrm{p}$ & INFN-LNS, Catania, Italy \\
2002 & $\mathrm{p}$ & Wakasa, Japan \\
\hline
\end{tabular}

9116

3927

1320

145

700

4646

318

738

1584

11414

3129

3766

486

220

920

2867

111

262

316

829

462

$1099+131$

930

2080

114

33

$\begin{array}{lll}2003 & \mathrm{p} & \text { Shizuoka, Japan } \\ 2004 & \mathrm{p} & \text { WPTC, Zibo, China } \\ 2006 & \mathrm{p} & \text { MD And., Houston, USA } \\ 2006 & \mathrm{p} & \text { FPTI, Jacksonville, USA }\end{array}$

At least three remarks can be made looking to this data : there is nearly the same number of centres in Europe (11), north America (8) and Japan (8), - the proton centres largely dominate, - the number of constructions is rapidly increasing these last few years.

This is confirmed in the table below which gives the expected year of first operation for hadrontherapy facilities in a commissioning phase, under construction or in a planning stage.

$\begin{array}{lll}2007 & \mathrm{p} & \text { RPTC, Munich, Germany } \\ 2007 / 08 & \mathrm{p} & \text { PSI, Villigen, Switzerland } \\ 2007 & \mathrm{p} & \text { NCC, Seoul, Korea } \\ 2007 & \mathrm{p}+\mathrm{i} & \text { HIT, Heidelberg, Germany } \\ 2007 & \mathrm{P}+\mathrm{i} & \text { CNAO, Pavia, Italy } \\ 2009 & \mathrm{p} & \text { UPenn, USA } \\ 2009 & \mathrm{p} & \text { WPE, Essen, Germany } \\ 2009 ? & \mathrm{p} & \text { iThemba Labs, South Africa } \\ 2009 ? & \mathrm{p} & \text { RPTC, Koeln, Germany } \\ 2010 ? & \mathrm{p} & \text { ICPO, Orsay, France } \\ 2010 ? & \mathrm{p} & \text { Trento, Italy } \\ 2011 ? & \mathrm{i} & \text { Gunma Univ, Japan } \\ 2011 ? & \mathrm{p} & \text { Chicago, USA } \\ 2011 ? & \mathrm{p}+\mathrm{i} & \text { PTC, Marburg, Germany } \\ 2011 ? & \mathrm{p}+\mathrm{i} & \text { ETOILE, Lyon, France } \\ 2011 ? & \mathrm{p}+\mathrm{i} & \text { Med-AUSTRON, Austria }\end{array}$

This non exhaustive list shows that - more than 16 centres will enter in operation over 5 years, - Europe largely dominates with 11 facilities (5 in Germany), - the proportion of ion facilities is growing fast, - as in the synchrotron light source domain, there is a switch form a first generation (parasitic use of the beam) to the second generation of dedicated facilities (PSI, ICPO for example), - a large majority of these new facilities ( $p$ and i) are or will be built as "turnkey facilities" by specialized Companies. (Rieneker PTC, Marburg, ETOILE...).

\section{MEDICAL BENEFIT OF HADRONTHERAPY}

\section{Particles versus Photons}

It is well known that the concentration of energy deposition of ions in a localized Bragg pick (Fig. 1) gives a better ballistic precision compared to photons. The different treatment must nevertheless be compared in term of better preservation of the healthy tissues and "Organ at Risk" in the tumor vicinity. The recent progress done with IMRT (Intensity Modulated Radio-Therapy), Cyber Knifes ... which compensate the ballistic drawback of the photons by a better geometric and quantitative dose 
distribution, as well as the fact that photons and protons have the same radiobiological efficiency, leads to a situation where there is no clear advantage of protons versus photons for most of the treatments.

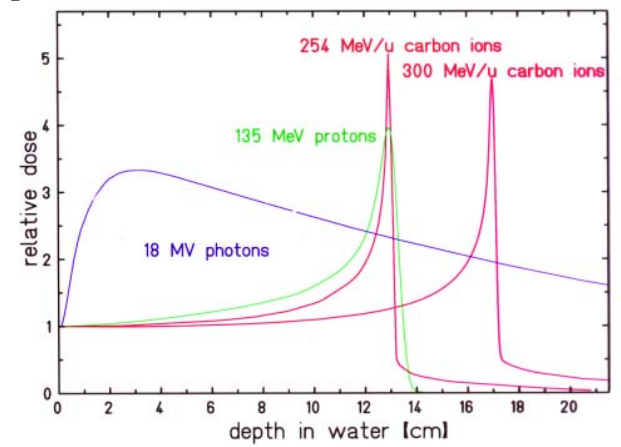

Fig. 1: Dose deposition of photons, protons and carbon ions in water.

\section{Carbons versus Protons}

Concerning the ballistic precision, carbon ions have the disadvantage of dose deposition after the Bragg pick due to the ion fragmentation (Fig. 2). Nevertheless, this drawback is largely compensated by a much lower radial diffraction (Fig. 3), a diffraction which penalizes protons for the treatment of deep-seated tumours.

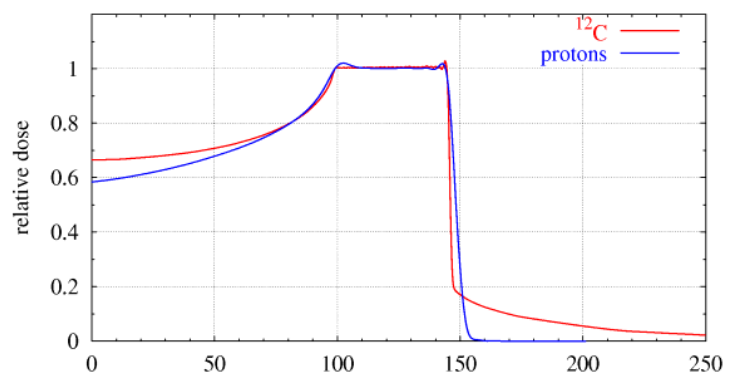

Fig. 2: Example of dose deposition of protons and carbon ions in water (Spread Out Bragg Pick).

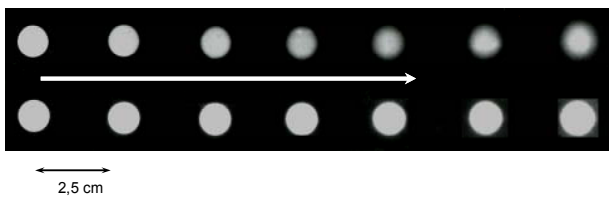

Fig. 3: Radial diffusion of protons (upper pictures) and carbon ions in water (E Blakeley LBL).

\section{Photons / Protons / Carbons medical benefits}

The decisive advantage of ions compared to photons and protons comes from their higher Linear Energy Transfer (LET) and Radio Biological Effectiveness (RBE). The RBE value is fixed at 1 for photons, it is around 1.1 for protons and reach $3.0 \ldots 5,0$ and above for carbon ions in the tumours depending of their composition. This property is illustrated on Fig. 4 which shows a clear advantage for the carbon ions in case of radio-resistant tumours representing approximately $10 \%$ of the total number of radiotherapies (meaning that the need is around 1 centre for 15 million inhabitants).

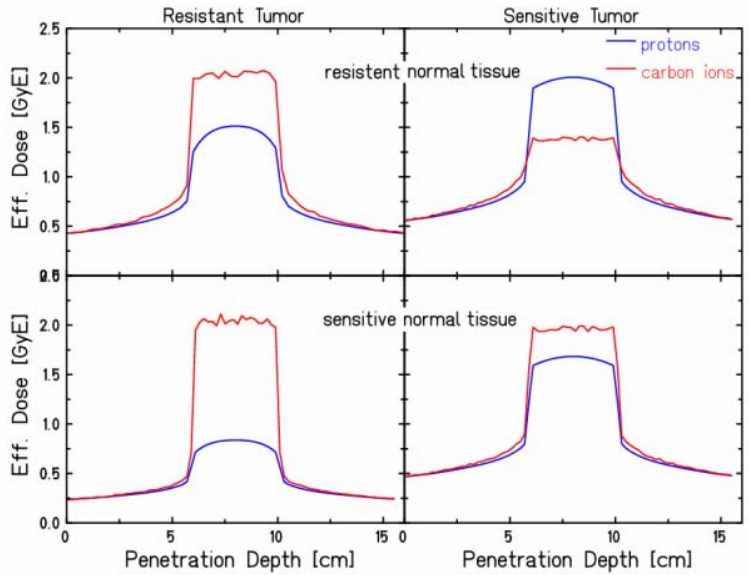

Fig. 4: Comparison of efficient dose deposition (Gy Equivalent) of protons and carbon ions for resistant and sensitive tumours and normal tissues surrounding the tumour (M. Scholz GSI).

\section{SOME HADRONTHERAPY FACILITIES IN EUROPE}

Looking to the list of hadrontherapy centers given in the first section of this paper, it is obvious that an exhaustive description of the European centers cannot be envisaged here. After the medical arguments developed in the previous sections, the choice has been made to focus our attention on the development of carbon therapy.

\section{Pioneer work at GSI}

With HIMAC in Japan, GSI is the place where a decisive pioneer work has been done for carbon-therapy. First of all, the GSI Biophisik Group has done the research works on the Relative Biological Effectiveness (RBE) of the ions and the links between RBE and DNA damages, repairing and non-repairing cells. The "LocalEffect-Model" (cell survival) has then been built and verified making in-vitro and in-vivo experiments. Finally, the RBE modelling has been validated and a Treatment Planning System (TPS) has been constructed.GSI makes also decisive technical developments to improve the quality of the treatments. The most famous is the raster scanning (spot-scanning) technique which allows a nearly perfect dose deposition in the tumour with an optimal preservation of the surrounding tissues (Fig 5). This "active" technique is more precise than the "passive" one based on the use of collimators and energy degraders. It has been use to treat more than 300 patient at GSI and is also used at PSI.

\section{HIT Heidelberg, Germany}

After a long period of technical work and discussions, the work done at GSI has lead to the construction of a dedicated heavy ion therapy centre at Heidelberg (Fig. 6). GSI made the accelerator, beam lines ( 2 horizontal) and rotating gantry designs (Fig. 7 and 8) [2,3]. The first turn in the synchrotron has been achieved in February this year, the commissioning of the facility in progress. 


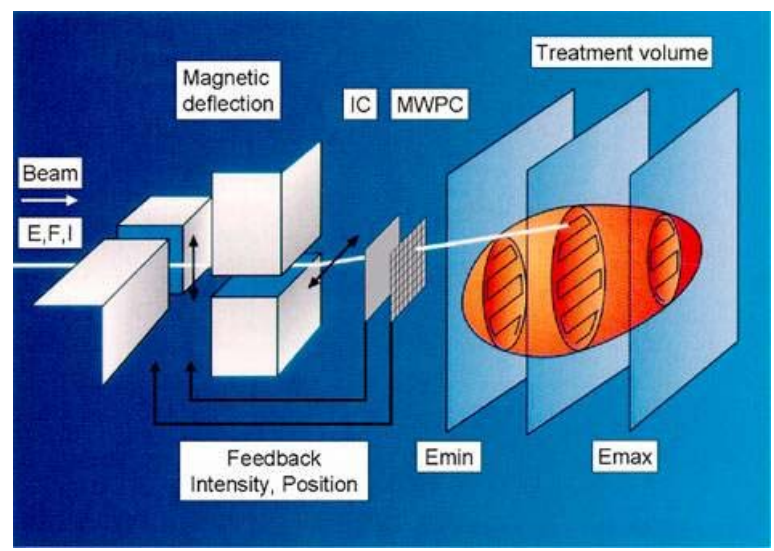

Fig. 5: The spot-scanning technique developed at GSI, schematic view of the hardware.

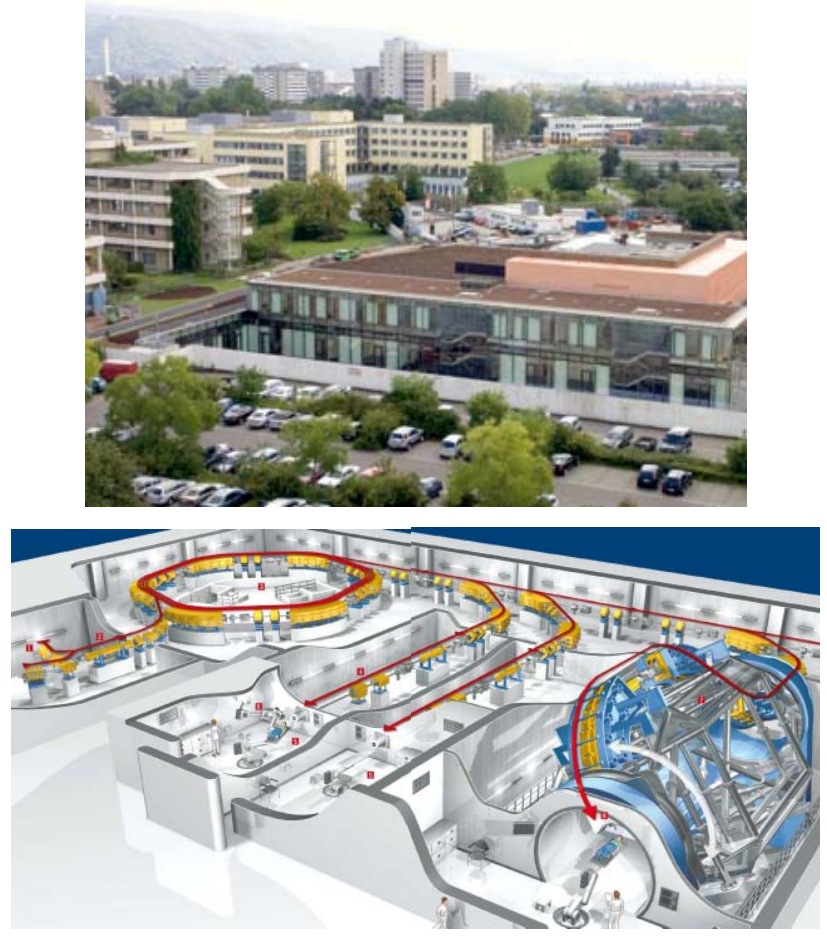

Fig. 6: HIT Heidelberg Centre, photo of the building (up) and schematic view of the injector, synchrotron, horizontal beam lines and rotating gantry (down).

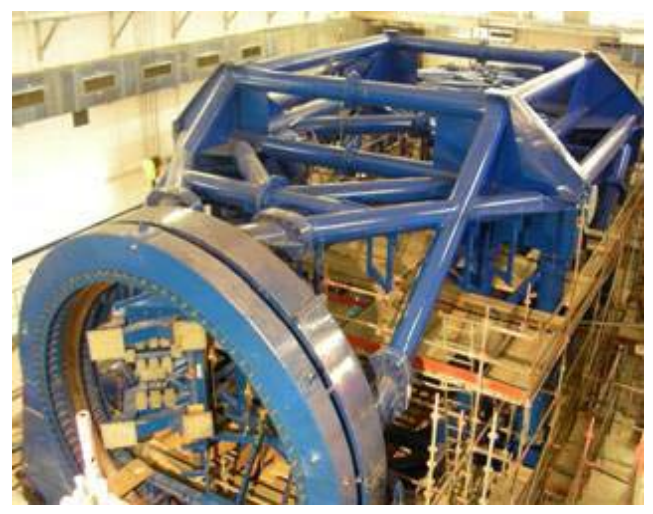

Fig. 7: Photo of the HIT Heidelberg gantry, the total rotating weight is $\approx 600$ tons, the weight of the last $90^{\circ}$ bending magnet is $\approx 60$ tons.

\section{CNAO, Pavia, Italy}

The realization of the Italian National Centre for Oncological Hadrontherapy (CNAO from the Italian acronym) started in September 2002 with the final design. The basic design of the accelerator and beam lines comes from the Proton-Ion Medical Machine Study (PIMMS) done at CERN from 1996 to 1999 with the TERA group who was the promoter of this project since 1992. This carbon facility (Fig. 8) is equipped with 3 treatment rooms in a first phase ( 2 with horizontal beams, 1 with horizontal and vertical beams) and a room for QA and experimental researches. The goal is to treat 3000 patients per year. The buildings construction is done to allow an extension housing a carbon rotating gantry.
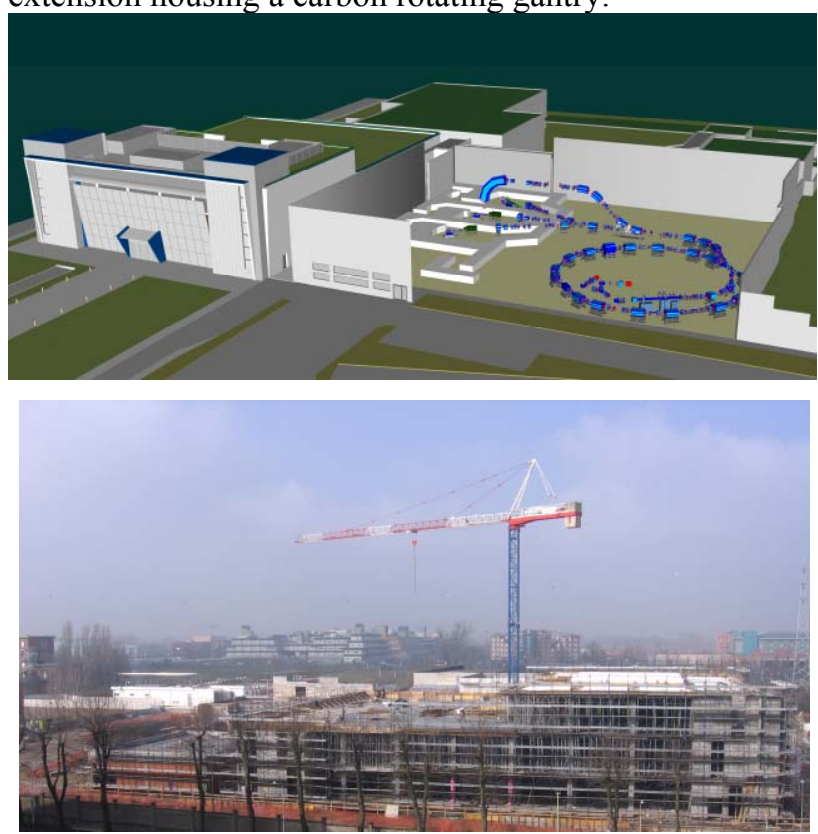

Fig. 8: CNAO (Pavia, Italy) carbon therapy Centre, schematic view with the synchrotron and beam lines (up) and photo of the building (February 2007, down).

\section{ETOILE, Lyon, France}

The construction of ETOILE, the French National Hadrontherapy Centre, has been approved by the French ministries of health and research in May 2006. This carbon therapy centre will be built in the framework of a Public - Private Partnership : - the Private Partner will finance and built the facility, - the facility will be the property of the Private Partner who will operate and maintain the facility (technical operation), - The medical team (Public Partner) will rent the beam time. The Private Partner selection will be made before end-2008.

\section{HADRONTHERAPY IS A BUSINESS}

Like radiotherapy... and therapy... hadrontherapy is now a business. The first table in the introduction shows that 15 "built by industry" protontherapy centres are running today (11 constructed by European companies, 4 by Japanese companies). The second table shows an acceleration of the phenomena, for protons but also for 
carbons (Marburg, ETOILE and MedAustron for example).

\section{$I B A$}

With 9 centers in operation and a large majority of the new orders, IBA is the world leader in protontherapy today. The offer is based on the IBA proteus 235 compact normal-conducting cyclotron (Fig. 9), horizontal beam lines and rotating gantries for passive treatments (an active scanning system is under study). IBA is also proposing a challenging carbon facility scheme based on a supraconducting isochronous cyclotron accelerating $\mathrm{Q} / \mathrm{M}=1 / 2$ ions at $400 \mathrm{MeV} / \mathrm{U}$ (Fig. 10).

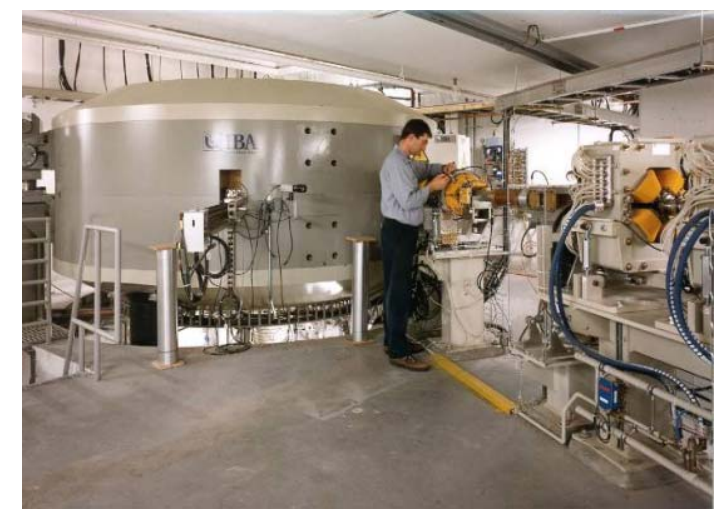

Fig. 9: IBA proteus 235 compact normal-conducting cyclotron.

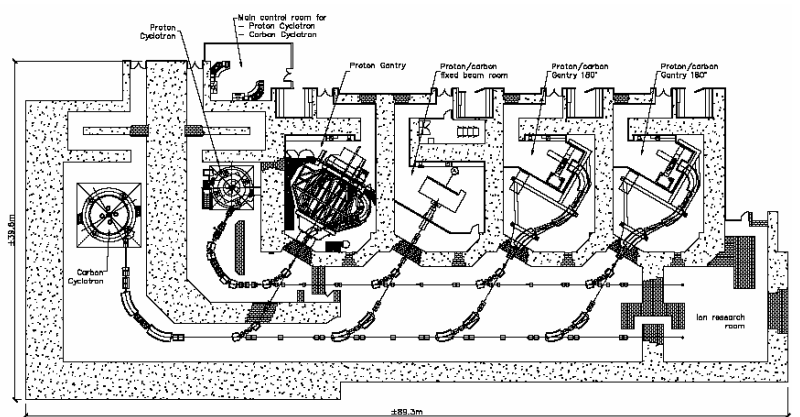

Fig. 10: IBA proposition of carbon therapy centre. From the left to the right: $400 \mathrm{MeV} / \mathrm{U}$ carbon superconducting cyclotron, proton cyclotron, proton gantry, horizontal fixed beam treatment room, two proton / carbon gantries and one research area.

\section{$A C C E L$}

Accel participates to the construction of the PROSCAN facility at PSI and Rinecker Proton Therapy Center (RPTC) in Munchen. These offers based on a very compact supraconducting cyclotron (Fig. 11) include the beam lines and the rotating gantries.

For the PROSCAN proton therapy centre at PSI (Fig. 12), the goal is to switch from the first generation (SINQ parasitic use) to a second generation with the ACCEL supraconducting cyclotron, new beam lines and a new gantry (the CPO in France is in a similar situation but IBA has been chosen). March 22, 2007, a PSI communicate announced that "Clinicians at the Paul Scherrer Institute in Switzerland have begun clinical treatments using the world's first commercial supraconducting cyclotron for routine medical use".

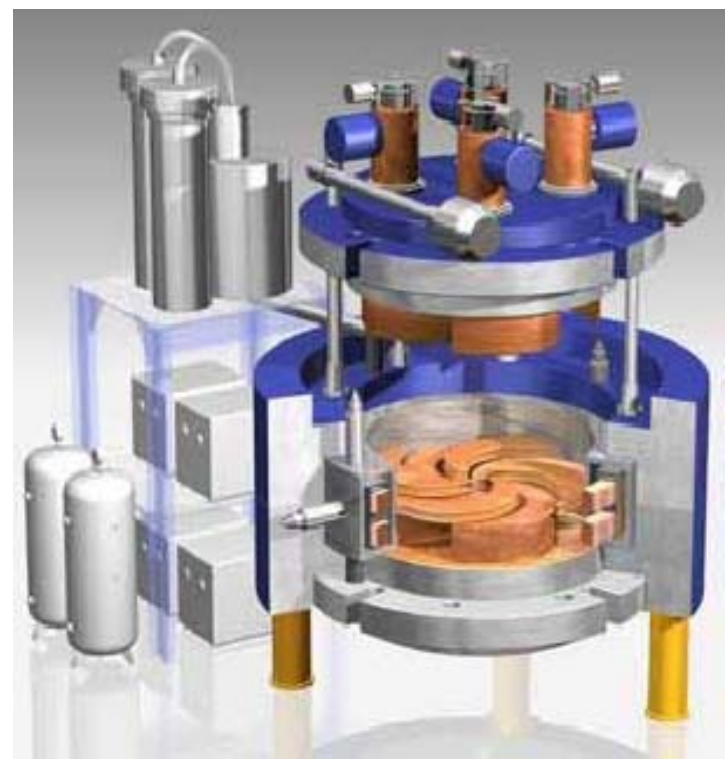

Fig. 11: Accel supraconducting cyclotron.

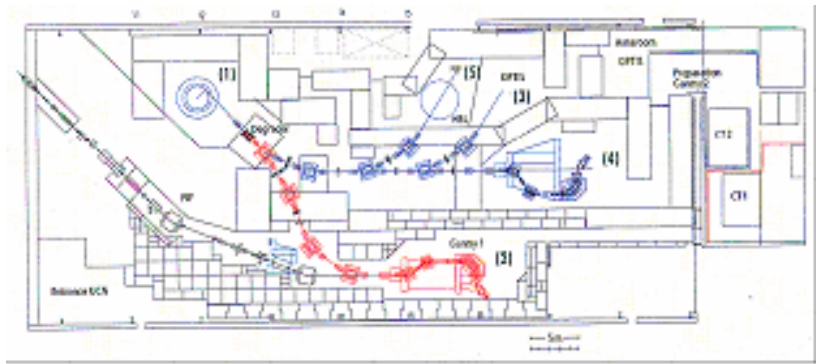

Fig. 12: Scheme of the PROSCAN facility at PSI.

The RPTC which is in the commissioning phase will work with 4 gantries and one horizontal fixed beam line, the present situation is the following:

- Extracted beam up to $1 \mu \mathrm{A}$

- Beam energy variable from 70 to $250 \mathrm{MeV}$

- Extraction efficiency of cyclotron $70 \%$

- Routine beam delivery to gantry room G1

- Scanning systems operable in G1

- Patient positioning / verification accepted by TÜV

- Software tools for G1 completed (incl. the TPS)

- Successful test of $\sim 1 \%$ dose conformity by technical expert of approving authority

ACCEL is part of Varian Medical Systems since the beginning of this year. The combination of Varian's expertise in image-guided radiation therapy (IGRT), treatment planning, information systems, clinical workflow, manufacturing, service and distribution with the ACCEL's proton therapy technology and expertise allows to offer a fully integrated proton treatment system from a single company.

\section{SIEMENS}

SIEMENS Medical Solutions has an industrial offer of turnkey proton plus carbon facilities thanks to a 
knowledge-transfer \& licenses from GSI (synchrotron and raster scanning technology, treatment optimization including the biological effects of ions, treatment planning software). For the construction of the new facilities, Danfysik is the exclusive supplier of accelerator systems for SIEMENS (from the sources up to the treatment rooms), they propose a standardized solution for proton and carbon therapy in one system.

But accelerator and beam lines are just one component of the hadrontherapy centre. The SIEMENS standardized solutions also include :

- Scanning and beam monitoring systems,

- Gantries, robotic tables for patient positioning in the treatment rooms,

- Patient transport systems,

- Imaging (diagnostic and position verification),

- Treatment Planning System,

- IT (oncology information system, administration, archiving...),

- Project management,

- Technical operation and maintenance.

The first turnkey facility built by SIEMENS is the Marburg carbon therapy centre. Fig. 13 shows an optimized use of the surfaces for the patient flow.

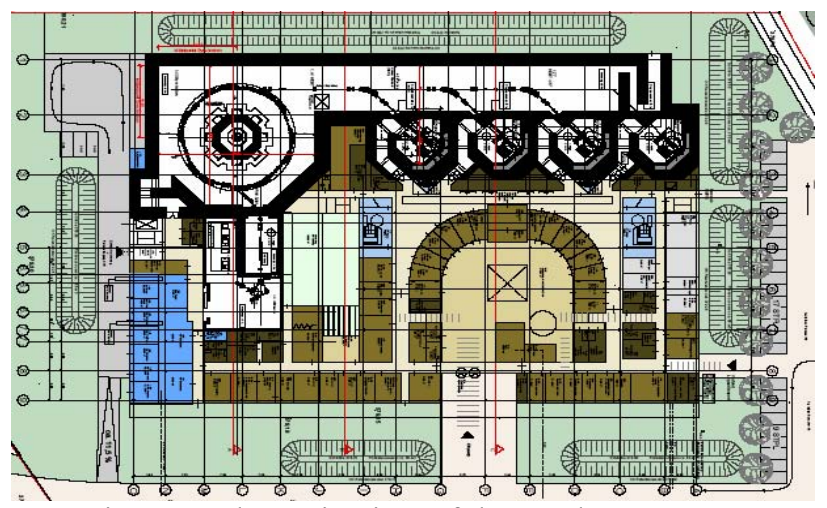

Fig. 13: Schematic view of the Marburg centre.

\section{SUMMARY}

Hadrontherapy is born thanks to the developments made in the field of fundamental physics (Pioneer work at Berkeley, Uppsalla... Fermi for Loma Linda... Chiba, GSI...). Today, the accelerator technologies are optimized in terms of performance and cost for nearly all the components and hadrontherapy has switched in the business domain with a hard industrial competition.

Electron, proton and heavy ion accelerators for medical applications (15 000 following Ugo Amaldi) are now built (and sometimes operated) by industry. Several companies have demonstrated the ability to built very performing accelerator turnkey facilities with medical assurance quality standards for the best price. Is it a lesson for the construction of accelerator facilities for fundamental research in the future?

The involvement of Academic Laboratories is then more and more complex, even if some groups are still getting some money to study exotic concepts with few chances to obtain better performances at lower cost. One of the exceptions could result from the work done on carbon gantries (Fig. 14) using superconducting magnets. The goal must be to decrease the cost, not to decrease the weight or the size !

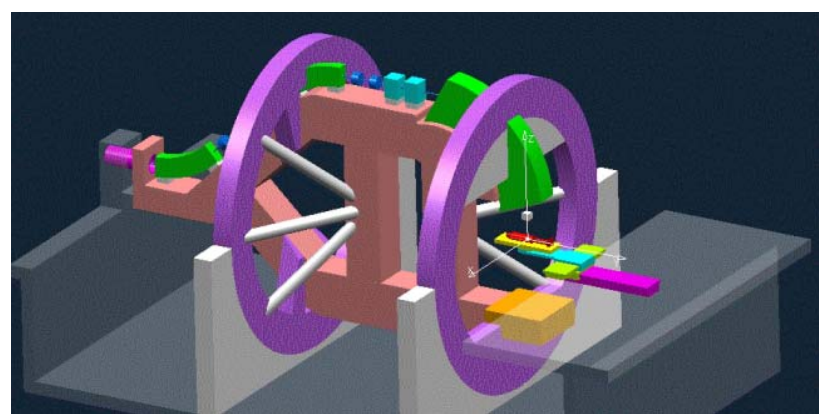

Fig. 14: Schematic scheme of a rotating gantry. How can we make it cheaper?

The highest R\&D priorities are not strictly related to accelerators, they deal with - the Treatment Planning Systems (error tolerance, better taking into account of the RBEs, Monte Carlo simulations), - fast and accurate beam monitoring systems, - ergonomic and accurate patient positioning system, - respiration gating (moving targets), dose homogeneity (repainting), - imaging and online dose monitoring (online PET for QA ...).

From the medical benefit of hadrontherapy point of view, protontherapy is in competition with the new photon technologies (new IMRT systems). The medical experts are not unanimous; it seems that active spot scanning protontherapy gives better results than MRT for several specific cases. On the other hand, the higher ballistic precision and RBE give a clear medical advantage to carbon for the treatment of radio-resistant tumours. This "market" is estimated to $\sim 10 \%$ of the total radiotherapy "market" (1 facility for $\sim 15 \mathrm{M}$ inhabitants).

Finally, impossible to end this paper without saying that hadrontherapy is the most motivating "application of accelerators". Physicists interested to go further in this domain must visit the PTCoG web site and follow the links... and attend the PTCoG meetings which are by far the most interesting on this topic.

\section{ACKNOWLEGEMENTS}

Many thanks to Gerhard Kraft and Wilma KraftWeyrather (GSI), Udo Weinrich (GSI - HIT), Sandro Rossi (CNAO), ETOILE and ASCLEPIOS teams members and to the HIMAC colleagues.

\section{REFERENCES}

[1] http://ptcog.web.psi.ch/

[2] U. Weinrich, "Gantry design for proton and carbon hadrontherapy facilities", TUYFI01, EPAC'06, Edinburgh, July 2006.

[3] S. Rossi, "Development in proton and light-ion therapy", FRYAPA01, EPAC'06, Edinburgh, July 2006. 